\title{
THE CLAIMS RESERVING PROBLEM IN NON-LIFE INSURANCE: SOME STRUCTURAL IDEAS
}

\author{
By Elja ArJas \\ University of Oulu, Finland
}

\begin{abstract}
We present some relatively simple structural ideas about how probabilistic modeling, and in particular, the modern theory of point processes and martingales, can be used in the estimation of claims reserves.
\end{abstract}

\section{INTRODUCTION}

The claims reserving problem, or the run off problem, has been studied rather extensively. The monograph by TAYLOR (1986) covers most of the developments so far, and, interestingly enough, creates a taxonomy to the models introduced. The booklet of VAN EEGHEN (1981) has a somewhat similar aim. Because of these recent surveys we do not intend to describe "the state of the art" in this area but confine ourselves to a few remarks.

There has been a clear tendency away from deterministic "accounting methods" into more descriptive probabilistic models. Early works in this direction were BüHLMANN et al. (1980), HACHEMEISTER (1980), LINNEMANN (1980) and REID (1981). Of more recent contributions we would like to mention particularly PENTIK ÄINEN and RANTALA (1986), and three papers dealing with unreported (IBNR) claims: NorbERG (1986), RobBIN (1986) and JEWELL (1987).

Most authors today tend to agree that there are important benefits from using structurally descriptive probabilistic models in insurance. However, there appears to be a new problem : With the increased realism of such models, many papers introduce, very early on, a long list of special assumptions and a correspondingly complicated notation. A reader may then not be able to see what ideas are really important and characteristic to the entire claims reserving problem, and what are less so, only serving to make the calculations more explicit. It would be more pleasant if the modeling could be started virtually without any assumptions, and then only adding assumptions as it becomes clear that advancing otherwise is difficult. We think that the modern theory of stochastic processes comes here to aid, and try to illustrate this in the following. We are mainly using "the martingale approach to point processes", as discussed e.g. in BRÉMAUd (1981) and KARR (1986). However, apart from some calculations towards the end, no previous knowledge of this theory is really needed to understand the paper. 
The emphasis of this paper is in the conceptual analysis of Section 2 and the structural results of Section 3. Section 4 provides an illustration of how the actual stochastic calculus, in a simple form, can be applied to obtain more explicit results.

We want to stress that this paper contains very little that could be called "new results": it is more important to us here how we arrive at them.

\section{CLAIMS, INFORMATION AND SETTLEMENT AS MARKED POINT PROCESSES}

Considering a fixed accident year, say the unit interval $(0,1]$, let the exact occurrence times of the accidents be $T_{1}^{*} \leq T_{2}^{*} \leq \ldots$ An accident which occurs at time $T_{i}^{*}$ is reported to the company after a random delay $D_{i}$ so that its reporting time is $R_{i}^{*}=T_{i}^{*}+D_{i}$. We denote the ordered reporting times (order statistics) by $T_{1}<T_{2}<\ldots$, assuming for simplicity that they are all different.

We follow the convention that the accidents are indexed according to the order in which they are reported to the company, i.e., the accident reported at $T_{i}$ is called "the $i^{\text {th }}$ accident". Because of the random delay in reporting this indexing is often different from the one that refers to the occurrence times.

In practice the number of accidents in a given year of occurrence is of course finite. We denote this (random) number by $N$. As a convention, we let the sequence $\left(T_{i}\right)$ be infinite but define $T_{N+1}=T_{N+2}=\ldots=\infty$.

Let us then assume that every time a new accident is reported to the company, this will be followed by a sequence of "handling times". These handlings could be times at which claim payments are paid, but also times at which the file concerning the accident is updated because of some arriving new information. Supposing that the $i^{\text {th }}$ accident has altogether $N_{i}$ handling times following its reporting, we denote them by

$$
T_{i}=T_{i 0}<T_{i 1}<T_{i 2}<\ldots<T_{i, N_{i}}
$$

Again, we let $T_{i, N_{i}+1}=T_{i, N_{i}+2}=\ldots=\infty$.

Next we need to specify the event that takes place at $T_{i j}$. If a payment is made then, we denote the amount paid by $X_{i j}$. If nothing is paid at $T_{i j}$ we simply let $X_{i j}=0$. Similarly, it is convenient to have a notation for the information which is used for updating the accident file. Let $I_{i 0}$ be the information which becomes available when the accident is reported, and let $I_{i j}$ be the new information which arrives at handling time $T_{i j}$. If there is no such information, we set $I_{i j}=\emptyset$, signalling " no new information". In particular we set $X_{i j}=0$ and $I_{i j}=\emptyset$ whenever $T_{i j}=\infty$.

Our analysis will not depend on what explicit form the variables $I_{i j}$ are thought to have. They could well be strings of letters and numbers, reflecting, for example, how the accident is classified by the company at time $T_{i j}$. $I_{i 0}$ will often determine what was the delay in reporting the $i^{\text {th }}$ accident. If further payments are made after the case was thought in the company to be closed, it is probably convenient to consider the arrival of the first such claim as a new reporting time, also initiating a new sequence of handlings. 
The above definitions give rise to a number of stochastic processes which are of interest in the claims reserving problem. The first definitions will be accident specific, after which we obtain the corresponding collective processes by simple summation.

We start by assigning the payments $X_{i j}$ to the handling times $T_{i j}$. In this way we arrive, for each $i$, at a sequence $\left(T_{i j}, X_{i j}\right)_{j \geq 0}$, where $T_{i}=T_{i 0} \leq T_{i 1} \leq \ldots$ (with strict inequalities if the variables are finite) and $X_{i j} \geq 0$. Thus $\left(T_{i i}, X_{i j}\right)_{j \geq 0}$ can be viewed as a marked point process (MPP) on the real line, with non-negative real "marks" $X_{i j}$. We call it the payment process. Equivalently, of course, we can consider the cumulative payment process $\left(X_{i}(t)\right)$ defined by

$$
X_{i}(t)=\sum_{\left\{j: T_{i j} \leq t\right\}} X_{i j}
$$

Clearly, $X_{i}(t)$ represents the total amount of payments (arising from accident i) made before time $t$. The function $t \mapsto X_{i}(t)$ is an increasing step function, with $X_{i}(t)=0$ for $t<T_{i}$ (= reporting time) and $X_{i}(t)$ approaching, as $t \rightarrow \infty$, the limit

$$
X_{i}(\infty)=\sum_{j \geq 0} X_{i j}
$$

which is the total compensation paid for the $i^{\text {th }}$ accident. Similarly,

$$
\begin{aligned}
U_{i}(t) & =X_{i}(\infty)-X_{i}(t) \\
& =\sum_{\left\{j: T_{i j}>t\right\}} X_{i j}
\end{aligned}
$$

represents the total liability at $t$ coming from future payments, with $U_{i}(t)=X_{i}(\infty)$ for $t<T_{i}$ and $U_{i}(t)$ decreasing stepwise to 0 as $t \rightarrow \infty$.

We remark here that, in order to keep this simple structure, we do not consider explicitly the effects of interest rate or inflation. This means, among other things, that the future claims must be expressed in standardized (deflated) currency.

Second, we can consider the sequence $\left(T_{i j}, I_{i j}\right)_{j \geq 0}$ and call it the information process for the $i^{\text {th }}$ accident. This, too, is an MPP, with mark $I_{i j}$ taking values in some conveniently defined set. As mentioned earlier the form of the marks is not restricted in any real way: It will suffice, for example, that there is a countable number of possible marks.

Our third MPP is obtained by combining the marks of the other two, into pairs $\left(X_{i j}, I_{i j}\right)$. We call $\left(T_{i j},\left(X_{i j}, I_{i j}\right)\right)_{j \geq 0}$ the settlement process of the $i^{\text {th }}$ accident. 
Considering finally all accidents collectively, we obtain the corresponding collective payment process, information process and settlement process by a simple summation (superposition) over the index $i$. However, we do not need a separate notation for these MPP's and will therefore confine ourselves to the cumulative payment process

$$
X .(t)=\sum_{i} X_{i}(t)
$$

and the liability process

$$
U \cdot(t) \sum_{i} U_{i}(t)
$$

Observe that it is not necessary to restrict the summation to indices $i$ satisfying $i \leq N$ because, unless this is satisfied, $X_{i}(t)=U_{i}(t)=0$ for all $t$.

\section{CLAIMS RESERVES AS A PREDICTION PROBLEM}

The estimation of the claims reserves can now be viewed as a prediction problem where, at a given time $t$ representing "the present", an assessment of the future payments is made on the basis of the available information. Most of our mathematical considerations do not depend on whether the assessment concerns the payments from an individual $i^{\text {th }}$ accident, or all accidents during the considered year of occurrence. Because of this we will often simply drop the subscript (" $i$ " or ".") from the notation. Thus, for example, $U(t)$ can be taken to be either the accident specific liability $U_{i}(t)$ or their sum $U .(t)$.

The role of the information process above is to provide a formal basis for the assessments made. This is done most conveniently in terms of histories, i.e., families of $\sigma$-fields in the considered probability space, which correspond to the knowledge of the values of the random variables generating them. In particular, we let the $\sigma$-field

$$
\mathscr{F}_{t}^{N}=\sigma\left\{\left(T_{i j}, I_{i j}, X_{i j}\right)_{i \geq 1, j \geq 0}: T_{i j} \leq t\right\}
$$

respresent the information carried by the pre- $t$ settlement process arising from all claims. (For background, see e.g. KARR (1986), Section 2.1). For completeness, we also allow for the possibility of having information which is exogenous to the settlements. Writing $\mathscr{\sigma}_{t}$ for such pre- $t$ information, we shall base the estimation of the future payments on the history $\left(f_{t}\right)$, with

$$
F_{t}=F_{t}^{N} \vee \epsilon_{t}
$$

In an obvious sense, the most complete assessment at time $t$ concerning $X(\infty)$, the total of paid claims, is provided by the conditional distribution

$$
\mu_{t}(\cdot)=P\left(X(\infty) \in \cdot \mid, z_{t}\right) .
$$


When $t$ varies, these conditional distributions form a so called prediction process $\left(\mu_{t}\right)$ (see e.g. Norros (1985)). Here, however, we restrict our attention to the first two moments of $\mu_{t}$. Assuming square integrability throughout this paper, we write

$$
M_{t}=E^{\prime \prime}(X(\infty)) \quad\left(=\int x \mu_{t}(d x)\right)
$$

and

$$
V_{t}=\operatorname{Var}^{\prime \prime}(X(\infty)) \quad\left(=\int x^{2} \mu_{t}(d x)-M_{t}^{2}\right)
$$

We now derive some fundamental properties of $\left(M_{t}\right)$ and $\left(V_{t}\right)$. From now on we also write $X_{t}$ and $U_{t}$ instead of $X(t)$ and $U(t)$.

Having introduced the idea that $F_{l}$ represents "information which the company has at time $t$ ", it is of course the case that the payments already made are, at least in principle, included in such knowledge. Formally this corresponds to the decomposition of $X_{\infty}$ into $X_{t}$ and $U_{t}$ (see (2.4)), i.e.,

$$
X_{\infty}=X_{t}+U_{t},
$$

where $X_{t}$ is determined from (i.e., $F_{t}$-measurable). Therefore, the $\left(F_{t}\right)$ based prediction of $X_{\infty}$ is equivalent to predicting $U_{t}$.

Conditional expectations. Let us first consider the expected values $M_{t}$. As a stochastic process, $\left(M_{t}\right)$ is easily seen to have the martingale-property: For any $t<u$,

$$
E^{\prime \prime}\left(M_{u}\right)=M_{t}
$$

Thus, since $M_{t}$ is an estimate of $X_{\infty}$ at time $t$ and $M_{u}$ is a corresponding updated estimate at a later time $u,(3.6)$ expresses the simple consistency principle:

(P1) "Current estimate of a later estimate, which is based on more information, is the same as the current estimate".

Another way to express the martingale property is to say that the estimates $\left(M_{t}\right)$ have no trend with respect to $t$.

Since $X_{t}$ is determined from $\sigma_{t}$ we clearly have

$$
M_{t}=X_{t}+E^{\prime \prime}\left(U_{t}\right) \underset{\text { def }}{=} X_{t}+m_{t} .
$$

Here, the estimated liability at $t$,

$$
m_{t}=E^{r_{t}}\left(U_{t}\right)
$$


is a supermartingale, with the "decreasing trend property"

$$
E^{\prime}\left(m_{u}\right) \leq m_{t} \quad \text { for } \quad t<u \text {. }
$$

This follows readily from the fact that the true liability $U_{t}$ is decreasing in time, as more and more of the claims are paid. Unfortunately such a monotonicity property is of little direct practical use because the process $\left(U_{t}\right)$ is unobservable: Only the differences $U_{u}-U_{t}=X_{t}-X_{u}$ can be observed, but not the actual values of $U_{u}$ or $U_{t}$.

The trend properties of $\left(M_{t}\right)$ and $\left(m_{t}\right)$ lead to a crude idea about how the reserve estimates should behave as functions of time. Considering them as a time series may therefore be useful. On the other hand, one has to remember that the (super)martingale property is quite weak and only concerns the $\left(\mathscr{F}_{t}\right)$-conditional expected values. Thus an apparently downward trend in an observed time series could be balanced by a rare but big jump upwards.

For a more refined analysis, it would be interesting to study $\left(M_{t}\right)$ in terms of its martingale integral representation (see e.g. BRÉMAUD (1981)). The key ingredient in that representation is the innovation gains process which determines how $\left(M_{t}\right)$ is updated in time when $\left(g_{t}\right)$ is observed. This theory is well understood. Unfortunately, however, actuaries seem to have very little idea about what properties the updating mechanism should realistically possess, and presently there is no detailed enough data to study the question statistically. Therefore, a more systematic research effort must wait.

It is instructive to still consider the differences

$$
M(t, u)=M_{u}-M_{t}, t<u .
$$

By the martingale property (3.6) we clearly have $E^{*}(M(t, u))=0$. Now, using the analogous notation $X(t, u)=X_{u}-X_{t}$ for the cumulative payments we easily find that

$$
M(t, u)=\left[X(t, u)-E^{\prime \prime}(X(t, u))\right]+\left[E^{\prime \prime \prime}\left(U_{u}\right)-E^{\prime \prime}\left(U_{u}\right)\right] .
$$

The first term on the right is the error in the estimate concerning payments in the time interval $(t, u]$. The second term, then, is the updating correction which is made to the estimated liability when the time of estimation changes from $t$ to $u$. Both terms have $F_{t}$-conditional expected value 0 . This suggests that it might be beneficial in practice to split the estmate into two parts: one that covers the time interval to the next update (typically a year) and another for times thereafter.

CONDITIONAL VARIANCES. The variances $V_{t}$ give rise to somewhat similar considerations. First observe that, since $X_{t}$ is determined by $\tau_{t}$, the variance $V_{t}$ defined in (3.4) satisfies

$$
V_{t}=\operatorname{Var}^{\prime}\left(U_{t}\right)=\operatorname{Var}^{\prime}(M(t, \infty))
$$


Thus, if the used estimation method produces also estimates of $V_{t}$, the observed oscillations in $\left(U_{t}\right)$ can be compared with the square root of $V_{t}$. (Warning: Do not expect normality in short time series!) Second, it is interesting to note that $\left(V_{t}\right)$ is a supermartingale as well, i.e.,

$$
E^{\prime \prime}\left(V_{u}\right) \leq V_{t} \quad \text { for } \quad t<u \text {. }
$$

This expresses the following intuitively plausible principle:

(P2) "Measured by the conditional variance, the estimates $M_{t}$ tend to become more accurate as time increases and more information becomes available".

To show that (3.10) holds, we first find that

$$
E^{\prime \prime}(M(t, u) X(u, \infty))=E^{\prime \prime}\left(M(t, u) E^{\prime \prime} M(u, \infty)\right)=0
$$

so that $M(t, u)$ and $M(u, \infty)$ are uncorrelated. This implies the well known additivity property ("Hattendorf's formula", e.g. GERBER (1979))

$$
\operatorname{Var}^{\prime}(M(t, \infty))=\operatorname{Var}^{\prime \prime}(M(t, u))+\operatorname{Var}^{\prime}(M(u, \infty)) .
$$

On the other hand,

$$
\operatorname{Var}^{\prime \prime}(M(u, \infty))=E^{\prime \prime}\left(\operatorname{Var}^{\prime \prime}\left(X_{\infty}\right)\right)=E^{\prime \prime}\left(V_{u}\right),
$$

so that (3.10) follows by combining (3.9), (3.11) and (3.12).

Remark. Recall the following well-known result which complements this picture: with respect to a quadratic loss function, the conditional expectation $M_{t}$ is the optimal estimate of $X(\infty)$. More precisely, for any estimate $\tilde{M}_{t}$ of $X_{\infty}$ which can be determined from $\tilde{r}_{t}$ (i.e., $\tilde{M}_{t}$ is $\tilde{r}_{t}$-measurable), the following inequality is satisfied:

$$
E^{\prime \prime}\left(\left(X_{\infty}-\tilde{M}_{t}\right)^{2}\right) \geq E^{\prime \prime}\left(\left(X_{\infty}-M_{t}\right)^{2}\right)\left(=V_{t}\right) .
$$

KNOWN AND UNKNOWN ACCIDENTS. Finally in this section we divide the collective estimate $m_{. t}=E^{\prime \prime}\left(U_{. t}\right)$ into two parts depending on whether the considered accidents are at time $t$ known (= reported, IBNER) or unknown (= not reported, IBNR).

Let the number of known (= reported) accidents at time $t$ be

$$
N_{t}=\sum_{i} 1_{\left\{T_{i} \leq t\right\}}
$$

The corresponding liability from future payments is then $\sum_{i \leq N_{t}} U_{i t}$. Since the events $\left\{T_{i} \leq t\right\}$ are determined by $\mathscr{F}_{t}$, the corresponding $\mathscr{T}_{t}$-conditional estimate is simply given by

$$
E^{\prime \prime}\left(\sum_{i \leq N_{i}} U_{i t}\right)=\sum_{i \leq N_{i}} E^{\prime \prime}\left(U_{i t}\right)=\sum_{i \leq N_{i}} m_{i t} .
$$


This formula expresses the intuitively obvious fact that the reserves corresponding to reported accidents could, at least in principle, be assessed individually.

If we are willing to make the assumption, which may not be completely realistic, that the liabilities $U_{i t}$ are uncorrelated across accidents given $\mathscr{r}_{l}$, we also have a corresponding equality for variances:

$$
\operatorname{Var}^{\prime \prime}\left(\sum_{i \leq N_{t}} U_{i t}\right)=\sum_{i \leq N_{t}} \operatorname{Var}^{\prime \prime} U_{i t}=\sum_{i \leq N_{t}} V_{i t} \text {. }
$$

Note that although the processes $\left(m_{i t}\right)$ and $\left(V_{i t}\right)$ were above found to be supermartingales, the processes defined by (3.15) and (3.16) do not have this property. This is because $N_{t}$ is increasing.

Considering then the unknown (IBNR) accidents, it is obvious that also their number $N-N_{t}$ is unknown (i.e., not determined by $N_{t}$ ) and therefore the liability estimate $E^{\prime \prime}\left(\sum_{i>N_{t}} U_{i t}\right)$ cannot be determined "termwise" as was done in (3.15). Therefore the estimate needs to be determined collectively for all IBNR-accidents, a task which we consider in the next section. The only qualitative property which we note here is that the process $\left(E^{\prime}\left(\sum_{i>N_{t}} U_{i t}\right)\right)$ is again a supermartingale. This is an easy consequence of the supermartingale property of $\left(m_{i t}\right)$, which was established above, and the fact that $N_{t}$ is increasing.

\section{AN ILLUSTRATION: THE ESTIMATION OF IBNR CLAIMS RESERVES}

We now illustrate, considering the IBNR claims reserves, how the mathematical apparatus of the stochastic calculus can be used to derive explicit estimates. But we are also forced to introduce some more assumptions in order to reach this goal.

For known accidents, the delays in the reporting times $T_{i}$ are only important in so far as they are thought to influence the distribution of the corresponding payment process. For unknown accidents the situation is completely different: For unknown accidents the only thing which is known is that if an $i^{\text {th }}$ accident occurred during the considered year and it is still unknown at time $t$, its reporting time $T_{i}$ exceeds $t$. (Recall the convention that $T_{i}=\infty$ for $i>N$ ). Therefore, it is impossible to estimate the IBNR reserves individually. A natural idea in this situation is to use the information which has been collected about other (i.e., known) accidents and hope that they would have enough in common with those still unknown. The problem resembles closely those in software reliability, where the aim is to estimate the unknown number of "bugs" remaining in the program. More generally, it is a state estimation or filtering problem. 
It is most convenient to formulate the "common elements" in terms of unobservable (latent) variables whose distribution is updated according to the information $F_{t} . F_{\text {, }}$ has thereby an indirect effect on the behaviour of IBNR claims. In the following we study the expected value and the variance of the IBNR liability. The presentation has much in common with JEWELL (1980, 1987), and RoBBIN (1986), and in particular NORBERG (1988).

Since the marked points belonging to the settlement process of an unknown accident are all "in the future", most considerations concerning the reserves will not change if the payments are assigned directly to the reporting time $T_{i}$. This is possible because we, as stated before, don't consider the effects of interest rate or inflation. This will simplify the notation to some extent. We therefore consider the MPP $\left(T_{i}, X_{i}\right)$, where $X_{i}=X_{i}(\infty)$ is the size of the claim caused by the $i^{\text {th }}$ accident. The corresponding counting process is $\left\{N_{t}(A) ; t \geq 0, A \subset R^{1}\right\}$, where

$$
N_{i}(A)=\sum_{i} 1_{\left\{T_{i} \leq t, X_{i} \in A\right\}}
$$

counts the number of accidents reported before $t$ and such that their liability $X_{i}$ is in the set $A$. (Note that $N_{t}(A)$ cannot in general be determined from $F_{t}$ since the $X_{i}$ 's counted before $t$ may also include payments made after time $t$. Also observe the connection to (3.14): $N_{t}=N_{t}\left(R^{1}\right)$ ).

For the purpose of using the apparatus of the stochastic calculus we start by writing the total liability from IBNR claims as an integral (pathwise):

$$
\sum_{i>N_{t}} U_{i t}=\sum_{\left\{i: T_{i}>t\right\}} X_{i}=\int_{s=t}^{\infty} \int_{x=0}^{\infty} x d N_{s}(d x)
$$

We also let

$$
\tilde{U}(t, u ; A)=\sum_{\left\{i: t<T_{i} \leq u, X_{i} \in A\right\}} X_{i}=\int_{s=t}^{u} \int_{x \in A} x d N_{s}(d x),
$$

so that $\sum_{i>N_{t}} U_{i t}=\tilde{U}\left(t, \infty ; R^{1}\right)$.

Adapting the idea from Norberg (1986) we now suppose that the above mentioned latent variables form a pair $(\Phi, \Theta)$ and are such that $\Phi$ can be viewed as a parameter of the distribution of the process $\left(N_{t}\right)$, formed by the reporting times, whereas $\Theta$ parametrizes the distribution of the claim sizes $\left(X_{i}\right)$. (Note that this simple model is "static" in the sense that the latent variables do not depend on time. This assumption could be relaxed, for example, by introducing an autoregressive scheme of state equations, as in the Kalman filter). There are no restrictions on the dimension of $(\Phi, \Theta)$. On the other hand, 
these parameters are assumed to be sufficient in the sense that if $\Phi$ and $\Theta$, together with some initial information $F_{0}$, were known, no information from $F_{t}$ would change the prediction concerning the IBNR claims after $t$. Thus the estimates of $\Phi$ and $\Theta$ which are obtained from $F_{t}$, or more exactly, their conditional distribution given $\mathscr{F}_{t}$, can be said to include "that part of $F_{t}$-information which is relevant in the IBNR-problem".

The formal expression of this idea is as follows. Fixing $t$ ("the present") we consider times $u \geq t$ and define

$$
\tilde{f}_{u}=\mathscr{F}_{0} \vee \sigma\{\Phi, \Theta\} \vee \sigma\left\{\left(T_{i}, X_{i}\right) ; t<T_{i} \leq u\right\}
$$

Thus $\tilde{f}_{\infty}$ represents the information contained collectively in $\tilde{f}_{0}$, the parameters $\Phi$ and $\Theta$, and all post- $t$ payments, cf. KARR (1986), Section 2.1. We then assume the conditional independence property

$$
\tilde{F}_{\infty} \coprod_{\gamma_{0} \vee \sigma\{\phi, \theta\}} \tilde{f}_{t},
$$

stating that $F_{t}$ is irrelevent for predicting the post- $t$ payments provided that $\tau_{0}$ and $(\Phi, \Theta)$ are known.

Let the $\left(\tilde{F}_{u}\right)$-intensity of counting process $\left(N_{u}(A)\right)_{u \geq t}$, be $\left(\tilde{\lambda}_{u}(A)\right)_{u \geq t}$, with $A \subset R^{1}$. The probabilistic interpretation of $\tilde{\lambda}_{u}(A)$ is that

$$
\tilde{\lambda}_{u}(A) d u=P\left(d N_{u}(A)=1 \mid \tilde{F}_{u^{-}}\right)=P\left(T_{i} \in d u, X_{i} \in A \mid \tilde{F}_{u^{-}}\right)
$$

on the interval $T_{i-1}<u \leq T_{i}$. On the other hand, $\tilde{\lambda}_{u}(A)$ can obviously be expressed as the product

$$
\tilde{\lambda}_{u}(A)=\bar{\lambda}_{u} \varphi_{u}(A)
$$

where $\bar{\lambda}_{u}=\tilde{\lambda}_{u}\left(R^{1}\right)$ and $\varphi_{u}(A) / \bar{\lambda}_{u}$ (cf. KARR (1986), Example 2.24). Here $\left(\bar{\lambda}_{u}\right)$ is the $\left(\tilde{F}_{u}\right)$-intensity of the counting process $\left(N_{u}\right)$, i.e., $\bar{\lambda}_{u} d u=$ $P\left(d N_{u}=1 \mid \tilde{f}_{u-}\right)=P\left(T_{i} \in d u \mid \tilde{f}_{u-}\right)$ for $T_{i-1}<u \leq T_{i}$, whereas $\varphi_{u}(A)$ can be interpreted as the conditional probability of $\left\{X_{i} \in A\right\}$ given $\tilde{F}_{u-}$ and that $T_{i}=u$.

It follows from (4.4) that the intensity $\left(\tilde{\lambda}_{u}(\cdot)\right)_{u>t}$ can be chosen to be $F_{0}$-measurable and parametrized by $(\Phi, \Theta)$. According to "the division of roles of $\Phi$ and $\Theta$ " we now assume that in fact $\bar{\lambda}_{u}$ in (4.6) is parametrized by $\Phi$, and $\varphi_{u}(\cdot)$ by $\Theta . \bar{\lambda}_{u}$ can then be expressed in the form $\bar{\lambda}_{u}=h(u ; \Phi)$, where, for fixed $\Phi, u \mapsto h(u ; \Phi)$ is $F_{0}$-measurable. This is only another way of saying that the reporting process $\left(N_{u}\right)$ is assumed to be a doubly stochastic (nonhomogeneous) Poisson process (or Cox process) with random parameter $\Phi$. 
Similarly, we assume that the claim size distributions $\varphi_{u}(\cdot)$ can be written as $\varphi_{u}(A)=F_{u}(A ; \Theta)$, where, for fixed $u$ and $\Theta, F_{u}(\cdot ; \Theta)$ is a distribution function on $R_{+}^{1}$. This, then, amounts to saying that, given $\Theta$ and the (unobserved) IBNR reporting times, the claim sizes $X_{i}$ are independent.

We now derive an expression for the expected IBNR-liability $E^{\prime \prime}\left(\sum_{i>N_{t}} U_{i t}\right)$.

First note that $\tilde{f}_{t}=\tilde{I}_{0} \vee \sigma(\Phi, \Theta)$. By a straightforward calculation we get that

$$
\begin{aligned}
E^{\tilde{z}_{t}}\left(\sum_{i>N_{t}} U_{i t}\right) & =E^{\tilde{x}_{1}}\left(\int_{u=t}^{\infty} \int_{x=0}^{\infty} x d N_{u}(d x)\right) \\
& =E^{\tilde{*}_{i}}\left(\int_{u=t}^{\infty} \int_{x=0}^{\infty} x \tilde{\lambda}_{u}(d x) d u\right) \\
& =\int_{u=t}^{\infty} h(u ; \Phi) \int_{x=0}^{\infty} x F_{u}(d x ; \Theta) d u \\
& =\int_{u=t}^{\infty} h(u ; \Phi) m_{u}(\Theta) d u,
\end{aligned}
$$

where $m_{u}(\Theta)$ is the mean

$$
m_{u}(\Theta)=\int_{x=0}^{\infty} x F_{u}(d x ; \Theta)
$$

(The equality $\left({ }^{*}\right)$ here is a simple consequence of the definition of $\left(\tilde{\lambda}_{u}\right)$; for a general result see e.g. KARR (1986, Theorem 2.22). On the other hand, because of the conditional independence (4.4), we have that

$$
E^{\tilde{r}}\left(\sum_{i>N_{i}} U_{i t}\right)=E^{\tilde{j}_{i}} \vee \tilde{i}_{i}\left(\sum_{i>N_{i}} U_{i t}\right)
$$

and therefore finaly

$$
E^{\prime \prime}\left(\sum_{i>N_{t}} U_{i t}\right)=\int_{u=t}^{\infty} E^{\prime \prime}\left(h(u ; \Phi) m_{u}(\Theta)\right) d u
$$

We consider some special cases at the end of this section.

Let us then go over to calculating the corresponding conditional variance expression $\operatorname{Var}^{\prime}\left(\sum_{i>N_{i}} U_{i t}\right)$. The calculation goes as follows. 


$$
\begin{aligned}
& \operatorname{Var}^{\tilde{r}_{1}}\left(\sum_{i>N_{t}} U_{i t}\right) \\
& =\operatorname{Var}^{\tilde{\tilde{F}_{t}}}\left(\int_{u=t}^{\infty} \int_{x=0}^{\infty} x d N_{u}(d x)\right)=E_{(*)}^{\tilde{*}}\left(\left\langle\tilde{U}\left(t, \cdot ; R^{1}\right)\right\rangle_{\infty}\right)
\end{aligned}
$$

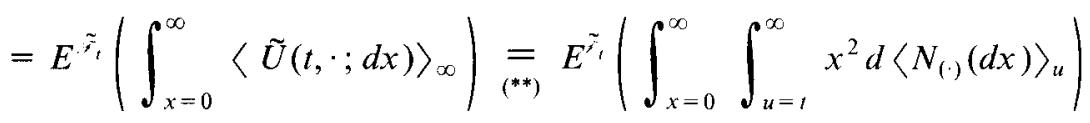

$$
\begin{aligned}
& =\tilde{E^{\prime}}\left(\int_{x=0}^{\infty} \int_{u=t}^{\infty} x^{2} \tilde{\lambda}_{u}(d x) d u\right)=\int_{u=t}^{\infty} h(u, \Phi) \int_{x=0}^{\infty} x^{2} F_{u}(d x ; \Theta) d u \\
& =\int_{u=t}^{\infty} h(u ; \Phi) m_{u}^{(2)}(\Theta) d u
\end{aligned}
$$

where $m_{u}^{(2)}(\Theta)$ is the second moment

$$
m_{u}^{(2)}(\Theta)=\int_{x=0}^{\infty} x^{2} F_{u}(d x ; \Theta)
$$

(Here $\left(\langle\cdot\rangle_{u}\right)$ is the predictable variation process, see e.g. KARR (1986), Appendix $\mathrm{B},\left(^{*}\right)$ is a direct consequence of the definition of this process, and (**) follows from Theorem B.12 in KARR (1986)). Therefore, and again using the conditional independence (4.4),

$$
\begin{aligned}
\text { (4.10) } & \operatorname{Var}^{\prime}\left(\sum_{i>N_{i}} U_{i t}\right)=E^{\prime \prime} \operatorname{Var}^{\tilde{*}}\left(\sum_{i>N_{i}} U_{i t}\right)+\operatorname{Var}^{\prime \prime} E^{\tilde{x}_{1}}\left(\sum_{i>N_{i}} U_{i t}\right) \\
= & \int_{u=t}^{\infty} E^{\prime \prime}\left(h(u ; \Phi) m_{u}^{(2)}(\Theta)\right) d u+\operatorname{Var}^{\prime \prime}\left(\int_{u=t}^{\infty} h(u ; \Phi) m_{u}(\Theta) d u\right) .
\end{aligned}
$$

The formulas (4.8) and (4.10) can be briefly summarized by saying that the conditional expectation and the conditional variance of the IBNR liability $\sum_{i>N_{t}} U_{i t}$ can be obtained if the following are known:

(i) the intensities $h(\cdot ; \Phi)$;

(ii) the first two moments of the distributions $F(\cdot ; \Theta)$, and

(iii) the conditional distribution of the latent variables $(\Phi, \Theta)$ given $r_{t}$.

Concerning (i), the common expression for $h(\cdot ; \Phi)$ (e.g. Rantala (1984)) is obtained by assuming that during the considered year ( = unit interval $(0,1])$ 
accidents occur according to the Poisson $(\Phi)$-process, and that the reporting delays $D_{i}$ are i.i.d. and distributed according to some known distribution $G(\cdot)$. Then it is easily seen that

$$
h(u ; \Phi)=\Phi\left[G(u)-G\left((u-1)^{+}\right)\right] .
$$

More generally, $\Phi$ can parametrize both the occurrence process and the distribution of the delays in the reporting, cf. JEwELL (1987).

The simplest case in (ii) is of course when only the number $N-N_{t}$ of future claims is considered, instead of the liability they cause. Then we can make the obvious convention that every $X_{i}=1$, giving $m_{u}(\Theta)=m_{u}^{(2)}(\Theta)=1$.

Requirement (iii), finally, strongly supports the use of the Bayesian paradigm. It is particularly appealing to use the Poisson-gamma conjugate distributions for the pair $\left(N_{t}, \Phi\right)$ since this makes the updating extremely simple (see Gerber (1979) and Norberg (1986)). Since deciding on claims reserves is a management decision, rather than a problem in science in which some physical constant needs to be determined, Bayesian arguments should not be a great deterrent to a practitioner. Choosing a reasonable prior for $(\Phi, \Theta)$ could be viewed as a good opportunity for an actuary to use, in a quantitative fashion, his experience and best hunches.

\section{ACKNOWLEDGEMENT}

Most of what I today know about insurance mathematics I have learned, during many long conversations, from JARMO JACOBSSON, of the Statistical Center of the Finnish Insurance Companies. I also appreciate the referees' careful reading of the paper.

This paper was read at the ASTIN Annual Meeting which was held in Helsinki in July 1988, in connection with the $23^{\text {rd }}$ International Congress of Actuaries.

\section{REFERENCES}

Brémaud, P. (1981) Point Processes and Queues: Martingale Dynamics. Springer-Verlag, Berlin. Búlumann, H., SchniePer, R. and Straub, E. (1980) Claims reserves in casualty insurance based on a probabilistic model. Bulletin of the Association of Swiss Actuaries, 21-45.

Gerber, H. U. (1979) An Introduction to Mathematical Risk Theory. S.S. Huebner Foundation of Insurance Education, University of Pennsylvania, Philadelphia.

Hachemeister, C.A. (1980) A stochastic model for loss reserving. Transactions of the $21^{s t}$ International Congress of Actuaries 1, 185-194.

JEWELL, W.S. (1980) Generalized models of insurance business. Report of Introduction. Transactions of the $21^{\text {st }}$ International Congress of Actuaries S, 87-141.

Jewell, W.S. (1987) Predicting IBNYR events and delays I. Continuous time. Department of Industrial Engineering and Operations Research, University of California at Berkeley, Research Report.

Karr, A. F. (1986) Point Processes and Their Statistical Inference. Dekker, New York.

Linnemann, P. (1980) A multiplicative model for loss reserves: A stochastic process approach. University of Copenhagen, Laboratory of Acuarial Mathematics, Working Paper No. 32.

Norberg, R. (1986) A contribution to modelling of IBNR claims. Scandinavian Actuarial Journal 1986, 155-203. 
Norros, I. (1985) Systems weakened by failures. Stochastic Processes Appl. 20, 181 - 196.

Pentikäinen, T. and Rantala, J. (1986) Run-off risk as a part of claims fluctuation. $A S T I N$ Bulletin 16, 113-147.

Rantala, J. (1984) An application of stochastic control theory to insurance business. Acta Universitatis Tamperensis A, 164 (Academic dissertation).

REID, D. H. (1981) A method of estimating outstanding claims in motor insurance with applications to experience rating. Colloque: Les mathématiques en Sciences Actuarielles-1981. Institut des Hautes Etudes de Belgique, Brussels, Belgjum, 275-289.

Robbin, 1. (1986) A Bayesian credibility formula for IBNR counts. Proceedings of the Cas. Actuarial Society Vol. LXXIII (1986), No. 139-140, 129-167 (with discussion).

TAYLOR, G. C. (1986) Claims Reserving in Non-Life Insurance. North-Holland, Amsterdam.

van Eeghen, J. (1981) Loss Reserving Methods. Surveys of Actuarial Studies No. 1, Nationale Nederlanden N.V.

\section{ElJa ArJas}

Department of Applied Mathematics and Statistics, University of Oulu, 90570 Oulu, Finland. 